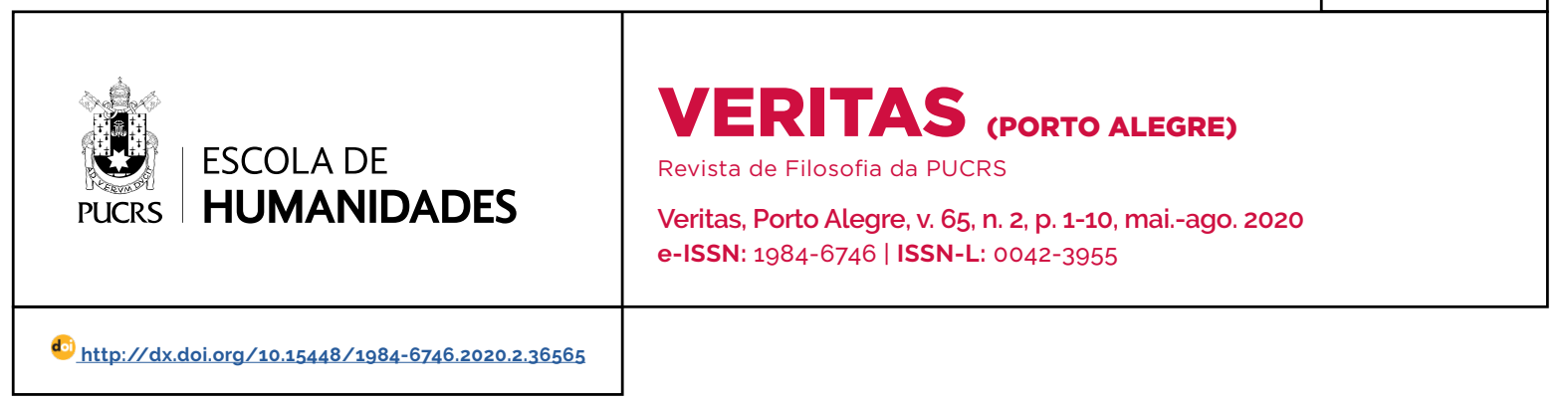

SEÇÃO: VARIA

\title{
Neurociência localizada: revendo diferenças de sexo/gênero em pesquisas sobre o cérebro
}

\author{
Located Neuroscience: reviewing sex/gender differences in brain research \\ Neurociencia localizada: revisando de las diferencias de sexo/género en la \\ investigación del cerebro
}

\section{Laura D. Guerim ${ }^{1}$ \\ orcid.org/0000-0002-3790-9053 \\ Laura.guerim@unilasalle.edu.br}

Recebido em: 30 nov. 2019.

Aprovado em: 18 fev. 2020

Publicado em: 28 jul. 2020

\section{(c) (1)}

Artigo está licenciado sob forma de uma licença Creative Commons Atribuição 4.0 Internacional.
Resumo: O objetivo deste trabalho é apresentar as criticas feitas por neurocientistas às pesquisas que buscam diferenças cognitivas entre homens e mulheres presentes no cérebro, principalmente, utilizando o respaldo da neuroimagem. Desde o início dos anos 2000 , a preocupação com a utilização da neurociência para justificar estereótipos de gênero e a falta de critério dos responsáveis para diferenciar as expressões "sexo" e "gênero" têm envolvido diversas neurocientistas no debate mais profundo entre natureza e cultura apresentado por essas pesquisas. Além disso, é imperativo pensarmos no impacto desses resultados, considerando que eles fornecem argumentos para os diversos discursos sociais e contribuem muito para o entendimento das habilidades humanas.

Palavras-chave: Neuriociência. Gênero. Sexo. Diferenças sexuais.

Abstract: The goal of this paper is to present critics made by neuroscientists to research that seeks differences presented in the brain between men and women, mainly using neuroimaging. Since early 2000s, the concern with the use of neuroscience to justify gender stereotypes and the lack of criteria from the researchers to differentiate the expressions "sex" and "gender" have involved many neuroscientists in the deeper nature and nurture debate presented by such researches. Besides, it is imperative to think about the impact of these results, considering that they provide arguments for the several social discourses and contribute a lot to the understanding of human abilities.

Keywords: Neuroscience. Gender. Sex. Sex differences.

Resumen: El objetivo de este trabajo es presentar las criticas hechas por los neurocientíficos a la investigación que busca diferencias cognitivas entre hombres y mujeres presentes en el cerebro, principalmente utilizando el apoyo de la neuroimagen. Desde principios de la década de 2000, la preocupación por el uso de la neurociencia para justificar los estereotipos de género y la falta de criterios por parte de los responsables de diferenciar las expresiones "sexo" y "género" han involucrado a varios neurocientíicos en el debate más profundo entre la naturaleza y la cultura presentada para estas búsquedas. Además, es imperativo pensar en el impacto de estos resultados, considerando que proporcionan argumentos para los diferentes discursos sociales y contribuyen mucho a la comprensión de las habilidades humanas.

Palabras clave: Neurociencia. Género. Sexo. Diferencias de sexo.

\section{Considerações introdutórias}

Em seu artigo mais clássico, Adina Roskies (2008) questiona de forma incisiva o papel dos exames de imagem em neurociência e compara os seus resultados e interpretações com aqueles discutidos 
em torno da fotografia convencional. Seria o exame de imagem uma foto do cérebro? Como os exames deveriam ser percebidos e interpretados? Essas são questões que norteiam o papel da neuroimagem no processo científico e nas consequências dos seus achados para além da comunidade científica.

O que vemos em exames de neuroimagem, como os realizados através de Imagem por Ressonância Magnética Funcional (FMRI) ou Tomografia Óptica Difusa (DOT), ${ }^{2}$ são diferenças no fluxo sanguíneo ativado ou não pela demanda de uma determinada região do cérebro, a partir de parâmetros estabelecidos pelo pesquisador, que proporcionam imagens funcionais ou estruturais do cérebro. Assim, diferente da fotografia convencional, as imagens obtidas através dessas técnicas são altamente dependentes de teorização e estão inferencialmente distantes do que de fato é gerado pelo cérebro. Roskies (2008) defende que o uso indiscriminado da neuroimagem como evidência de alterações fiéis e fixas do funcionamento do cérebro é perigoso, principalmente quando lidamos com o público leigo, pois, nas palavras de Letitia Meynell (2012, p. 12 tradução nossa), essas imagens "facilitam a apresentação de abstrações construidas como particularidades concretas". ${ }^{3}$

Em consonância com essa ideia de construção, muitas neurocientistas têm argumentado que boa parte dos estudos envolvendo a busca por diferenças acaba por reafirmar estereótipos, em vez de questioná-los. Em um dos seus livros mais recentes, The Gendered Brain, Gina Rippon (2019) avalia as diversas pesquisas que reafirmam através de "evidências científicas" estereótipos como o de que as mulheres são mais emotivas, inclusive por razões homonais (EPSTEIN, 2018), e os homens gostam mais de sexo (BRIZENDINE, 2006). Partindo da observação de Gustave Le Bon, no século XIX, de que as mulheres são inferiores aos homens, e chegando às polêmicas afirmações recentes de um funcionário da Google e do reitor da Universidade de Harvard, a autora desmistifica crenças estereotipadas de gênero que são refletidas nas grandes pesquisas em neurociência e desmascara a posição parcial da mídia em propagar os estudos de resultados errôneos e nunca corrigi-los para o grande público.

$O$ atual discurso neurocientífico focado principalmente nas diferenças entre homens e mulheres contribui fortemente para o debate sexo/gênero. Diversas pesquisas apontam que homens possuem mais facilidade para as ciências exatas e que as mulheres têm mais habilidades emocionais ou são mais empáticas. Homens utilizam mais regiões corticais frontais do cérebro do que mulheres, tornando-os mais racionais, e mulheres, desde muito cedo, têm dificuldades com o aprendizado da matemática. Essas habilidades, supostamente inatas, justificam a menor presença das mulheres como destaques em pesquisas das áreas de ciência, tecnologia, engenharia e matemática (Science, Technology, Engineering, Mathematics) e maior em profissões como psicologia e serviço social.

A partir do início dos anos 2000, diversas pesquisadoras das neurociências começaram a se preocupar com o fato de que essas pesquisas, mais do que questionar os estereótipos de gênero, acabavam por reafirmá-los e justificálos em nome da biologia. Revisões sistemáticas recentes mostram que, quando comparando grupos maiores de homens e mulheres, as diferenças presentes no cérebro são maiores intragrupos do que entre-grupos (ELSE-QUEST; HYDE; LINN, 2010; JORDAN-YOUNG, 2011). OU seja, a diferença encontrada entre homens e mulheres é menor do que as encontradas entre os homens e entre as mulheres quando se estuda um grupo maior de individuos, e não os grupos de 24 ou 27 individuos de cada sexo, como no caso das pesquisas que apontam diferenças. Ao questionar um pesquisador sobre isso, Robyn Bluhm (RIPPON, 2019, p. 118, tradução nossa) obteve o seguinte comentário: "se as diferenças

\footnotetext{
2 A maior parte das pesquisas em neurociência que são criticadas neste texto e em Gendered Brain (Rippon, 2019) utilizam desses exames de imagem.

Do original: [...] it facilitates the presentation of constructed abstractions as concrete particulars.
} 
de gênero (tipicamente) não aparecem em estudos de reação emocional, como vamos explicar o consenso de que existe diferença de gênero nas respostas emocionais?". ${ }^{4}$ Questionar esse consenso não parece ser uma possibilidade.

Além disso, as pesquisas que buscam diferenças entre homens e mulheres não apresentam diferenciação entre sexo e gênero. Para esses conceitos, os pesquisadores parecem não utilizar o mesmo cuidado criterioso que utilizam para outros (NIKOLEYCZIK, 2012). Sexo e gênero têm sido diferenciados pelas feministas desde a metade do século XX (BEAUVOIR, 2009; HOOKS, 2014). Para as pesquisadoras das neurociências de hoje, é imperativo esclarecer essa diferença nas pesquisas científicas atuais. Se sexo diz respeito aos aspectos biológicos das diferenças entre homens e mulheres, gênero dá conta de explicar as diferenças socioculturais entre os mesmos, que variam de acordo com a época e o contexto social (JOAN SCOTT, 1995). O que a neurociência tem feito ao ignorar a diferença entre esses dois conceitos é apresentar uma justificativa biológica para uma diferença social. Felizmente, novas pesquisas têm conseguido mostrar o quão social são essas diferenças, como veremos adiante.

Assim, este trabalho apresentará os principais argumentos de pesquisadores em neurociência na crítica às diferenças de sexo, além de pesquisas mais recentes que comprovam o viés social dessas diferenças e apontará para algumas soluções éticas no desenvolvimento de novas pesquisas na área.

\section{Gênero, sexo e neurosexismo}

É impossivel começar a explicar a construção de uma crítica à neurociência da diferença sexual sem explicitar a diferença entre sexo e gênero. Só assim será possivel entender o termo "neurosexismo", cunhado pela psicóloga Cordelia Fine (2008).

Sexo e gênero significam coisas distintas de acordo com cada teoria feminista. A proposta aqui não é trazer todas elas, mas sim apresentar em que ponto essa distinção faz diferença para as pesquisas na área da saúde, principalmente na neurociência. Apesar de os dois termos estarem relacionados, no sentido de surgirem um a partir do outro (gênero a partir de sexo biológico), eles possuem uma diferença importante. Sexo compreende os aspectos biológicos de homens e mulheres, as diferenças claramente observáveis entre as genitálias e demais características físicas, além dos cromossomos. Gênero compreende os aspectos socioculturais que são distintos entre homens e mulheres, como papéis sociais e comportamento.

Gayle Rubin (1975, p. 165, tradução nossa) explicitou bem essa diferença ao chamar essa relação entre os conceitos de "sistema sexo/ gênero" e dizer que ele é "[...] um conjunto de arranjos onde o material biológico cru do sexo humano e da procriação é formado pela intervenção humana e social".5 Assim, è a partir do sexo biológico apresentado pelo indivíduo ao nascer que uma série de significados será atribuida à sua identidade, transformando uma característica biológica em uma posição social.

Nesse sentido, homens e mulheres apresentam diferenças em diversos atributos físicos sim, mas principalmente nos sociais, considerando que não existe gênero fora do contexto social. Se vivemos em um mundo generificado, é impossivel supor que, em concordância com Butler (2003, p. 37),

[...] a discussão sobre identidade deveria ser
anterior à discussão sobre identidade de gê-
nero, simplesmente porque as pessoas só
se tornam inteligiveis ao ter seu gênero em
conformidade com os padrões reconheciveis
de inteligibilidade de gênero.

No estudo do cérebro, a preocupação em diferenciar "sexo" e "gênero" surgiu também a partir dos estudos sobre neuroplasticidade. Considerando a capacidade do cérebro de se adaptar a diferentes situações, de aprender novas habilidades e formar novas memórias - assim como novos neurônios -, além da compreensão

\footnotetext{
4 Do original: If gender differences (typically) fail to emerge in studies of emotional reactivity, how are we to explain the widespread consensus that there are gender differences in emotional responding?

5 Do original: a set of arrangements by which the biological raw material of human sex and procreation is shaped by human, social intervention.
} 
de que o córtex só está plenamente formado após os vinte anos de idade, é possivel imaginar a influência de aspectos culturais na estruturação morfofisiológica do cérebro. Anelis Kaiser e colaboradores (2009) ressaltam justamente essa influência quando dizem que

[...] diferenças de sexo no cérebro não são evidências de uma dimensão puramente material, mas refletem comportamentos generificados, aprendidos e incorporados em um contexto social. Uma vez incorporadas no cérebro, as diferenças de gênero tornam-se parte de nossa biologia cerebral.

Simone de Beauvoir (2009, p. 28) nota questão semelhante ao escrever que as "discriminações sociais que parecem insignificantes de fora e cujas repercussões morais e intelectuais são tão profundas na mulher que podem parecer ter suas raízes numa natureza original". Assim, natureza e cultura influenciam-se mutuamente e, mais importante, afetam sistemas biológicos, tais como o cérebro (ANNE FAUSTO-STERLING, 2000).

Jesse Prinz (2004, p. 132, tradução nossa) também escreve sobre isso, salientando estereótipos bem conhecidos que dizem respeito à superficialidade feminina e a maneira de se vestir. O filósofo diz que

o que consideramos masculino ou feminino parece ser parcialmente determinado pela cultura. Na cultura ocidental, a feminilidade está associada com sintomas tão arbitrários quanto vestidos, amor por flores, unhas feitas e a cor rosa. De forma ainda mais perturbadora, a feminilidade está associada à submissão, bons modos e frivolidade. ${ }^{6}$

Existe uma tendência atual em substituir o termo "sexo" pelo termo "gênero" em diferentes áreas da saúde (HAIG, 2004). Grosso modo, o termo "sexo" deveria dar conta das diferenças realmente biológicas (como as reprodutivas) entre homens e mulheres, enquanto o termo "gênero" deveria fazer referência às diferenças que não são necessariamente biológicas e como essas diferenças (comportamentais, por exemplo) influenciam a saúde, inclusive o seu acesso.
No estudo do cérebro, podemos estudar os componentes neurais envolvidos na regulação dos ciclos hormonais, como o eixo hipotálamohipófise, onde diferenças entre homens e mulheres estão presentes e são um tanto óbvias, na medida em que a regulação hormonal é bem diferente entre os sexos (mulheres menstruam, têm a capacidade de engravidar etc.). E, também, podemos estudar os aspectos cognitivos do cérebro, as funções executivas, memória, motricidade, planejamento e decisões morais. Nesse caso, não existe um preditor biológico capaz de justificar diferenças como as observadas nos estudos que as buscam.

Cordelia Fine foi uma das primeiras pesquisadoras a identificar esse viés em estudos nas neurociências. Ao utilizar o termo "neurosexismo", Fine (2008, 2010, 2013) apontou para a forma como as diversas pesquisas que utilizam neuroimagem apresentam uma diferença de sexo "extensiva, funcionalmente significativa e fixa, apoiando de forma implícita uma perspectiva essencialista de gênero" (2013, p. 369). Ainda, a pesquisadora mostra que, ao afirmarem, através de resultados falso-positivos as diferenças entre os sexos, e ignorarem a característica plástica do cérebro, essas pesquisas apresentam interpretações funcionais não fundamentadas em termos científicos e consistentes com estereótipos de gênero (2013).

\section{A construção da crítica}

É a partir da identificação do neurosexismo que diversas outras pesquisadoras irão desenvolver suas críticas em relação às interpretações das diferenças de sexo e suas consequências. Catherine Vidal (2012) retoma Paul Broca e ressalta o poder do estereótipo, ao deixar claro que ele acreditava na desconexão entre tamanho de cérebro e inteligência quando afirmou que "[...] as mulheres são um pouco menos inteligentes que o homem. Portanto, pode-se concluir que o cérebro menor da mulher pode ser explicado

\footnotetext{
6 Do original: what we consider masculine or feminine seems to be partially determined by culture. In Western culture, femininity is associated with such arbitrary symptoms as dresses, love of flowers, manicured nails, and the color pink. More disturbingly, femininity has been associated with submissiveness, manners and frivolity.
} 
pela sua inferioridade tanto em tamanho quanto em inteligência"(2012, p. 296, tradução nossa). Vidal (2012, p. 296) afirma que a "ideologia era mais forte do que a objetividade científica". Se tamanho fosse importante, a baleia azul, com seu cérebro de mais de $7 \mathrm{~kg}$, dominaria o mundo.

Em 2008, após a quebra da bolsa e consequente crise econômica mundial, Coates e Herbert, da Universidade de Cambridge, fizeram um experimento com 17 homens que trabalhavam na bolsa de valores. O objetivo do estudo era relacionar os niveis de testosterona com o comportamento econômico. Os resultados mostraram uma correlação positiva entre niveis altos de testosterona, risco financeiro assumido e o retorno financeiro. Uma brincadeira foi feita com o fato de que se o Lehman Brothers fosse Lehman Sisters, talvez o mercado não tivesse estourado. No caso dessa pesquisa, é muito dificil prever a concentração de testosterona no cérebro a partir da medida de testosterona obtida através da saliva. E essa evidência é uma correlação que não apresenta um real efeito causal.

Assim, uma pesquisa metodologicamente mais rigorosa (ZETHRAEUS et al., 2009), investigou a relação causal entre a concentração de hormônios sexuais e comportamentos de risco em 200 mulheres no periodo pós-menopausa. Os resultados mostraram que os niveis de testosterona e estrógeno não apresentaram efeitos significativos nos testes comportamentais.

Retomando Rippon (2019), falar em um cérebro feminino (female brain) é ressaltar diferenças causadas por fatores socioculturais. A autora critica o livro de Louann Brizendine. The Female Brain (2006) (inclusive o filme baseado no livro está disponivel no serviço de assinatura de streaming Netflix), afirmando que Brizendine não traz os dados corretos dos estudos que referencia, e apresenta dados já refutados ou inexistentes. Uma das "evidências" apresentadas por Brizendine é a de que as mulheres falam mais do que os homens. Ela diz em seu livro que as áreas do cérebro responsáveis pela linguagem são maiores nas mulheres e que elas usam cerca de 20 mil palavras por dia, enquanto os homens usam apenas 7 mil. Rippon mostra, então, que um pesquisador, ao conferir esses dados, não encontrou a pesquisa original e, ainda, descobriu que, utilizando uma base de dados de conversações britânicas, as mulheres na verdade falam em média um pouco menos de 9 mil palavras, enquanto os homens falam um pouco mais de 6 mil. Números bem diferentes daqueles citados por Brizendine. Outro equivoco foi apresentado em relação à empatia. Para Brizendine, o cérebro masculino tem menor capacidade de ser empático que o feminino. Ao procurar essas referências, Rippon não encontrou nenhuma evidência capaz de sustentar tal afirmação.

Livros como o de Brizendine formam uma categoria que Rippon chama de Neurotrash, ou neurolixo, criticando essa tendência de acrescentar o termo "neuro" em qualquer palavra. São livros que utilizam pesquisas de forma errônea para propagar crenças estereotipadas de gênero. Escolha o seu estereótipo favorito dentre as obras a seguir: Os homens são de Marte e as mulheres são de Vênus; Por que homens não escutam e mulheres não sabem ler mapas; Por que os homens mentem e as mulheres choram; e, ainda, Por que os homens gostam de linhas retas e as mulheres de poá. O problema dessa categoria é sua forte influência na mídia e seu apelo ao público leigo. Apesar disso, cada vez mais os neurocientistas, bem como alguns jornalistas, estão se posicionando contra essa tendência e criticando o poder da neuroimagem de transformar uma informação relativamente incompleta em verdade incontestável.

Uma das abordagens mais comuns para explicar diferenças entre os sexos presentes no cérebro é um paradigma conhecido como "hardwiring". A teoria, defendida por importantes pesquisadores, afirma que a influência de hormônios sexuais em determinados momentos do desenvolvimento do cérebro altera a morfofisiologia do mesmo e é a responsável pelas diferenças encontradas entre os sexos (BAO; SWAAB, 2010; BARON-COHEN, 2003; CAHILL, 2006; ELLIOT, 2009; HINES, 2004). Em um artigo na revista Neuroethics, Rebecca 
Jordan-Young e Raffaella I. Rumiati (2011) afirmam que não existe razão para não assumir que as diferenças observadas em diversas pesquisas não são frutos, ao menos em grande parte inclusive considerando a localização cortical dessas diferenças -, da distinta socialização de homens e mulheres. As autoras terminam o artigo citando Steven Rose e colegas (2009. p. 120, tradução nossa), que apontam que "[...] em uma sociedade onde o racismo e o sexismo não existem, as questões sobre os brancos e os homens serem mais ou menos inteligentes que os negros e as mulheres, não teriam apenas pouco significado, elas simplesmente não existiriam"?

Isabelle Dussauge e Anelis Kaiser (2012, p. 140, tradução nossa) resumem essas questões muito bem quando dizem que

nos estudos neurocientíicos revisados aqui, nós testemunhamos o desaparecimento de tudo que não está contido no corpo individual; nem as interações com o mundo, os imaginários culturais, os processos de criação de significado, tampouco as relações de poder possuem teórica permissão de deixar marcas no cérebro e/ou em suas funções; e não são consideradas partes relevantes das condições experimentais. ${ }^{8}$

\section{As comprovações necessárias}

Diversas pesquisas recentes têm conseguido mostrar o papel da socialização na construção dessas diferenças cognitivas observadas em homens e mulheres. É o caso do estudo de Bian, Leslie e Cimpian (SCIENCE, 2017). Nessa pesquisa, ao testar o estereótipo de que homens têm mais capacidades intelectuais do que mulheres, associando-os à genialidade e ao brilhantismo, os pesquisadores apresentaram os resultados de quatro estudos. O primeiro estudo examinou o desenvolvimento desse estereótipo ao longo do tempo em 96 crianças com idades entre $5 \mathrm{e}$ 7 anos (note que a raça e a classe social dessas crianças não moderaram significativamente os resultados obtidos). Além disso, esse estudo examinou também a percepção das crianças em relação ao quanto homens e mulheres parecem ser agradáveis. Segundo os autores, esses dois traços (brilhantismo e agradabilidade) se relacionam com gênero de formas diferentes (a saber, homens são brilhantes e mulheres agradáveis). Na medida em que as noções culturais de gênero são assimiladas, as crianças devem apresentar diferenças nas respostas. Os resultados sugerem que as ideias que as crianças possuem sobre brilhantismo mudam rapidamente entre os 5 e os 7 anos. Aos 5 anos, as crianças associam brilhantismo ao seu próprio gênero, mas as meninas de 6 a 7 anos fazem menos essa associação do que meninos. $O$ estereótipo associando mulheres à agradabilidade segue o mesmo caminho, onde os meninos associam cada vez menos o traço com seu próprio gênero.

No segundo estudo, agora com 144 crianças, meninas de 5 a 7 anos selecionaram igualmente outras meninas como as que possuiam maiores notas. No entanto, essas meninas não relacionaram as melhores notas com brilhantismo. Os autores concluem que a noção de brilhantismo não está necessariamente relacionada à percepção de quem tira melhores notas, mas sim às atitudes dos professores e ao viés do estereótipo de gênero. No estudo três, os pesquisadores investigaram a relação entre a noção de brilhantismo e os interesses das crianças. Aqui, os meninos, associando brilhantismo ao seu próprio gênero, mostraram maior interesse por atividades que envolviam a inteligência mais do que a persistência, sendo o oposto observado em meninas.

No quarto estudo, os pesquisadores compararam o interesse de meninos e meninas por atividades de crianças "muito, muito inteligentes". Entre os meninos e meninas de 5 anos não foi observada diferença no interesse pela atividade. Já entre as crianças mais velhas,

\footnotetext{
Do original: In a society in which racism and sexism were absent, the questions of whether whites or men were more or less intelligent than blacks and women would not merely be meaningless - they would not even be asked.

8 Do original: In the neuroscientific studies reviewed here, we witness the disappearance of everything which is not contained within the individual body; neither lived interactions with the world, cultural imaginaries, processes of meaning-making nor power relations are theoretically allowed to leave marks on the brain and/or its function; nor are they considered as relevant parts of the experimental conditions.
} 
de 6 e 7 anos, observou-se que as meninas se interessavam menos por esse tipo de atividade. No final das contas, os autores concluem que "muitas crianças assimilam a ideia de brilhantismo como uma qualidade masculina desde muito cedo"9 (BIAN; LESLIE; CIMPIAN, 2017, p. 391, tradução nossa) e que "esse estereótipo começa a moldar o interesse da criança assim que é adquirido e portanto é possivel que reduza a variedade de carreiras que essa criança um dia irá contemplar".10 (BIAN; LESLIE; CIMPIAN, 2017. p. 391, tradução nossa).

Em um estudo sobre a crença em suas próprias habilidades em matemática, Perez-Felkner, Nix e Thomas (2017), concluíram que, mesmo quando apresentam as mesmas notas que os meninos na disciplina de matemática, meninas adolescentes se percebem menos capazes de resolver problemas matemáticos mais desafiadores. Essa construção social da capacidade reduzida das mulheres nesse campo pode ser uma das razões pelas quais mulheres seguem menos as carreiras nas áreas de física, engenharia, matemática e ciências da computação.

Esses são alguns exemplos de pesquisas recentes que buscam derrubar os estereótipos que acabam perpetuando a desigualdade entre homens e mulheres nas diversas esferas da sociedade. É imperativo considerar que, para além das críticas às pesquisas que conservam intacto o status quo, precisamos pensar nas diferentes formas em que uma metodologia mais ética é capaz de resolver esse problema. As pesquisadoras envolvidas nas críticas também propõem novas abordagens para antigos problemas e os cuidados que podemos ter ao interpretar dados em pesquisas nas neurociências.

\section{Neuroética}

O poder discursivo das pesquisas em neurociências, respaldado sempre pelas belas imagens dos exames de Ressonância Magnética Funcional, nos trazem a preocupação sobre o que está sendo dito pelas pesquisas e como esses resultados se apresentam para o público leigo. Aqui, especificamente para a questão de gênero, apresento algumas propostas para atingirmos mais rapidamente a solução de tamanho problema.

Em 2002, após a conferência "Neuroethics: Mapping the Field", William Safire definiu neuroética como "o exame do que é certo e errado, bom e mau, sobre o tratamento, aperfeiçoamento, invasão indesejada e manipulação preocupante do cérebro" ([2002], tradução nossa). ${ }^{11}$ Também nesse ano, Roskies publicou um comentário na revista Neuron, intitulado "Neuroethics for the New Millennium". Nele, a autora divide o campo em duas partes: uma responsável pela ética da neurociência e a outra pela neurociência da ética. A primeira parte compreende as questões éticas relacionadas ao desenho experimental e execução das pesquisas em neurociência (ética da prática), e a avaliação do impacto dos resultados nas esferas sociais, éticas e legais (ética das implicações da neurociência). A segunda parte compreende o estudo neurocientífico de noções filosóficas que concernem a ética, como autocontrole, livre-arbítrio e intenção.

Roskies (2002) explica que a ética das implicações da neurociência é a área realmente nova do campo e que ela deve investigar as consequências do entendimento do funcionamento do cérebro para a sociedade. A autora diz que "avanços na neurociência têm o potencial de criar e solucionar inequidades sociais graves" e que "a forma como usamos nosso conhecimento, molda a nossa sociedade"12 (ROSKIES, 2002, p. 1, tradução nossa). Ela também atenta para o uso do conhecimento gerado pela neurociência ao indagar (ROSKIES, 2002, p. 1, tradução nossa):

como, ao entender mais e mais sobre como o cérebro controla o comportamento e as causas

\footnotetext{
9 Do original: Many children assimilate the idea that brilliance is a male quality at a young age

10 Do original: This stereotype begins to shape children's interests as soon as it is acquired and is thus likely to narrow the range of careers they will one day contemplate.

11 Do original: the examination of what is right and wrong, good and bad about the treatment of, perfection of, or unwelcome invasion of and worrisome manipulation of the human brain.

12 Do original: Advances in neuroscience have the potential to create, and to remedy, serious social inequities. How we use our knowledge will shape our society.
} 
das disfunções mentais, nós vamos conciliar esse novo conhecimento com as estruturas sociais que permitem o bom funcionamento da nossa sociedade? ${ }^{13}$.

Assim, podemos pensar nas muitas formas discutidas aqui, sobre como devemos tomar cuidado ao interpretar pesquisas que buscam diferenças de gênero e acabam, portanto, reforçando estereótipos e mantendo a inequidade. É fundamental buscarmos soluções éticas para o tratamento das pesquisas e seus consequentes resultados.

Nikoleyczik (2012, p. 242, tradução nossa), buscando apontar para a importância do uso dos estudos feministas e de gênero nas pesquisas em neurociência, diz que

integrar o conhecimento sobre gênero significa aumentar a consciência das complexidades e diferenciações de gênero/sexo e incluir muitas ou diferentes concepções de gênero/sexo nos projetos de pesquisa. ${ }^{14}$

A autora ainda apresenta algumas propostas para solucionar os problemas éticos decorrentes da ignorância do aspecto social da construção de sexo/gênero. São elas:

a) definir os conceitos de sexo/gênero e como eles se aplicam de forma teórica e experimental;

b) utilizar os estudos de gênero e a neuroética feminista, por exemplo, nas interpretações dos resultados e, assim, assumir a responsabilidade pelas implicações sociais da pesquisa;

c) utilizar a linguagem correta nas publicações decorrentes da pesquisa; e

d) cuidar ao generalizar resultados.

Springer, Stellman e Jordan-Young (2012) propõem algumas orientações para a pesquisa sobre sexo/gênero na área da saúde, principalmente quando ela não se relaciona com aspectos reprodutivos de homens e mulheres, necessitando, assim, de considerações sociais ou biossociais sobre diferenças de sexo/gênero. Entre as orientações, vale destacar, especificamente para a área da neurociência, as seguintes: 1) desenvolvimento, operacionalização e o teste de hipóteses alternativas que incluam caminhos sociais e biossociais; 2) atentar para questões de significância estatística e tamanho amostral; e 3) cuidado em interpretar e apresentar os resultados.

\section{Considerações finais}

Após apresentar uma das principais criticas às pesquisas em neurociências atuais, penso que ainda temos um longo caminho pela frente. É claro que as mudanças irão começar na academia. Poucas soluções abordam a importância da formação inicial na área médica, como uma fase importante para o aprendizado do desenvolvimento de pesquisas éticas ou da importância das mesmas enquanto formadores de conhecimento que irão impactar de forma substancial a nossa sociedade. Tornar artigos sobre essas pesquisas acessiveis em lingua portuguesa, como é o caso aqui, apresenta para nossos jovens pesquisadores um dos problemas urgentes da área a serem solucionados.

É importante ressaltar que este artigo discorre sobre gênero sem incluir raça e classe social no debate. Cada vez mais, percebemos a importância de discussões interseccionais que dêem conta da solução dos problemas na área da saúde e em como essa necessita conversar com os conhecimentos da área das ciências humanas. Ainda, quando falamos de homens e mulheres e a presença ou não de diferenças neurais entre eles nas pesquisas apresentadas aqui, estamos falando de homens e mulheres cisgênero. Os estudos que investigam diferenças entre pessoas cisgênero e transgênero acabam perpetuando estereótipos da mesma forma, mas as implicações políticas e de saúde dessas pesquisas ainda precisam ser profundamente analisadas para que sejam criticadas (o que certamente deverão ser).

Assim, o objetivo deste estudo foi trazer, de maneira inicial, os principais tópicos discutidos

13 Do original: How, as we learn more and more about how the brain controls behavior and the causes of mental dysfunction, are we to reconcile this new knowledge with the social structures that allow our society to run more or less smoothly?

14 Do original: Integrating gender knowledge means improving awareness of the complexities and differentiations of gender/sex and including several or different conceptions of gender/sex into one research project. 
hoje nas críticas sobre a forma como a neurociência têm abordado diferenças de sexo/ gênero. Percebemos que essas críticas estão bem fundamentadas e que mais pesquisas que consigam demonstrar a influência dos aspectos sociais na formação do cérebro precisam ser desenvolvidas. Além disso, é importante lembrar que, ao utilizar o conceito de neuroplasticidade e considerar as pesquisas mais recentes sobre gênero e habilidades em matemática, as palavras de Rippon fazem muito sentido: "um mundo generificado produzirá um cérebro generificado"15 (2019, p. 17, tradução nossa).

\section{Referências}

BAO, Ai-Min; SWAAB, Dick F. Sex differences in the brain, behavior, and neuropsychiatric disorders. Neuroscientist, [s. l.], v. 16, n. 5, p. 550-565, 2010. DOI: https://doi. org/10.1177/1073858410377005

BARON-COHEN, Simon. The Essential Difference: men, women and the extreme male brain. Londres: Allen Lane, 2003

BEAUVOIR, Simone de. O Segundo Sexo. Rio de Janeiro: Nova Fronteira, 2009

BIAN, Lin; LESLIE, Sarah Jane; CIMPIAN, Andrei. Gender stereotypes about intellectual ability emerge early and influence children's interests. Science, [s. l.], v. 355, n. 6323, p. 389-391, 2017. DOI: https://doi.org/10.1126/ science.aah6524.

BLUHM, Roberta. Self-Fulfilling Prophecies: the Influence of Gender Stereotypes on Functional Neuroimaging Research on Emotion. Hypatia, [s. l.], v. 28, p. 870-886, 2013. DOI: https://doi.org/10.1111/j. 1527-2001.2012.01311.X

BLUM, Deborah. Winter of discontent: Is the hot affair between Neuroscience and Science Journalism cooling down? Undark, Massachusetts, ano 2012, n. 3.

BRIZENDINE, Louann. The Female Brain. Nova lorque: Morgan Road, 2006.

BUTLER, Judith. Problema de Gênero: Feminismo e subversão da identidade. São Paulo: Civilização Brasileira, 2003

CAHILL, Larry. Why sex matter for neuroscience. Nature Reviews in Neuroscience, [s. L.], v. 7, n. 6, p. 477-484, 2006. DOI: https://doi.org/10.1038/nrn1909.

DUSSAUGE, Isabelle; KAISER, Anelis. Re-Queering the Brain. In: NEUROFEMINISM: ISSUES AT THE INTERSECTION OF FEMINIST THEORY AND COGNITIVE SCIENCE. Londres: Palgrave Macmillan, 2012. DOI: https://doi. org/10.1057/9780230368385_7.
ELIOT, Lise. Pink Brain, Blue Brain: How small differences grow into troublesome gaps - and what we can do about it. Nova lorque: Houghton Mifflin Harcourt, 2009.

EPSTEIN, Randi Hutter. Aroused: The History of Hormones and How They Control Just About Everything Nova lorque: WW Norton, 2018.

FAUSTO-STERLING, Anne. Sexing the body: Gender Politics and the Construction of Reality. Nova Iorque: Basic Books, 2000

FINE, Cordelia. Will working mothers' brains explode? The popular New Genre of Neurosexism. Neuroethics, [s. l.], v. 1, p. 79-82, 2008. DOI: https://doi.org/10.1007/ S12152-007-9004-2.

FINE, Cordelia. Delusions of Gender: How our minds, society, and neurosexism create difference. Nova lorque: W.W. Norton, 2010.

FINE, Cordelia. Is there neurosexism in Functional Neuroimaging investigations of sex difference? Neuroethics, [s. l.], v. 6, p. 369-409, 2013. DOI: https://doi.org/10.1007/ s12152-012-9169-1.

HAIG, David. The inexorable rise of gender and the decline of sex: social change in academic titles, 1945-2001. Archives of Sexual Behavior, [s. l.], v. 33. p. 87-96, 2004. DOI: https://doi.org/10.1023/B:ASEB. $0000014323.56281 .0 d$

HINES, Melissa. Brain Gender. Oxford: Oxford University Press, 2004

HOOKS, bell. Feminism is for everybody. Nova lorque: Routledge. 2014. DOI: https://doi. org/10.4324/9781315743189.

JORDAN-YOUNG, Rebecca. Brainstorm: The flaws in the science of sex differences. Cambridge: Harvard University Press, 2011.

KAISER, Anelis; HALLER, Sven; SCHMITZ, Sigrid; NITS$\mathrm{CH}$, Cordula. On sex/gender related similarities and differences in fMRI language research. Brain Research Reviews, [s. l.], v. 61, n. 2, p. 49-59, 2009. DOI: https:// doi.org/10.1016/j.brainresrev.2009.03.005.

MEYNELL, Letitia. The Politics of Pictured Reality: Locating the object from Nowhere in fMRI. In: BLUHM, Robyn; JACOBSON, Anne Jaap; MAIBOM, Heidi Lene (org.). Neurofeminism: issues at the intersection of feminist theory and cognitive science. Londres: Palgrave Macmillan, 2012. p. 12

NIKOLEYCZIK, Katrin. Towards Diffractive Transdisciplinarity: Integrating Gender Knowledge into the Practice of Neuroscientific Research. Neuroethics, Is. l.], v. 5, p. 231-245, 2012. DOI: https://doi.org/10.1007/ S12152-011-9135-3.

PEREZ-FELKNER, Lara; Nix, Samantha; THOMAS, Kirby. Gendered Pathways: How Mathematics Ability Beliefs Shape Secondary and Postsecondary Course and Degree Field Choices. Frontiers in Psychology, [s. l.], v. 8, p. 1-11, 2017. DOI: https://doi.org/10.3389/ fpsyg.2017.00386. 
POOLE, Steven. Your brain on pseudoscience: the rise of popular neurobollocks. New Statesman, Londres, 2012.

PRINZ, Jesse. Gut Reactions: a perceptual theory of emotions. Oxford: Oxford University Press, 2004.

QUART, Alissa. Neuroscience: under attack. New York Times, Nova lorque, 2012.

ELSE-QUEST, Nicole M.; HYDE, Janet Shibley; LINN, Marcia C. Cross-sectional Patterns of Gender Differences in Mathematics: A Meta-Analysis. Psychological Bulletin, [s. L.], v. 136, n. 1, p. 103, 2010. DOI: https://doi. org/10.1037/a0018053.

SCOTT, Joan. Gênero: uma categoria útil de análise histórica. Educação e Realidade, [s. l.], v. 20, n. 2, p. 71-99, 1995.

RIPPON, Gina. The Gendered Brain: The new neuroscience that shatters the myth of the female brain. Londres: The Bodley Head, 2019.

ROSE, Steven; CECl, Stephen; WILLIAMS, Wendy M. Should Scientists Study race and IQ? No: Science and Society do not benefit. Nature, [s. l.], v. 457, p. 786-788, 2009. DOI: https://doi.org/10.1038/457786a.

ROSKIES, Adina. Neuroethics for the New Millennium. Neuron, [s. l.], v. 35, p. 21-23, 2002. DOI : https://doi. org/10.1016/So896-6273(02)00763-8.

ROSKIES, Adina. Neuroimaging and inferential distance. Neuroethics, [s. l.], v. 1, p. 19-30, 2008. DOI: https://doi. org/10.1007/s12152-007-9003-3.

RUBIN, Gayle. The Traffic in Women: Notes on the 'Political Economy' of Sex. In: REITER (ed.). Toward an Anthropology of Women. New York: Monthly Review Press, 1975.

SAFIRE, William. 2002. Stanford Encyclopedia of Philosophy. Disponivel em: https://plato.stanford.edu/ entries/neuroethics/\#NeurSociJust.

SPRINGER, Kristen W:; STELLMAN, Jeanne Mager; JORDAN-YOUNG, Rebecca M. Beyond a catalogue of differences: A theoretical frame and good practices guidelines for researching sex/gender in human health. Social Sciences and Medicine, [s. l.], v. 74, p. 1817-1824, 2012. DOI: https://doi.org/10.1016/j.socscimed.2011.05.033.

VIDAL, Catherine. The Sexed Brain: Between Science and Ideology. Neuroethics, [s. l.], v. 5, p. 295-303, 2012. DOI: https://doi.org/10.1007/s12152-011-9121-9.

ZETHRAEUS, Niklas; KOCOSKA-MARAS, Ljiljana; ELLINGSEN, Tore; VON SCHOULTZ, Bo; HIRSCHBERG, Angelica Lindén; JOHANNESSON, Magnus. A randomized trial of the effect of estrogen and testosterone on economic behavior. Proceedings of the National Academy of Sciences of the United States of America, [s. L.], v. 106, p. 6525-6538, 2009. DOI: https://doi.org/10.1073/ pnas. 0812757106 .

\section{Laura D. Guerim}

Doutora em Clínica Médica pela Pontifícia Universidade Católica do Rio Grande do Sul (PUCRS, Porto Alegre, RS, Brasil). Pós-doutoranda no PPG em Memória Social e Bens Culturais Universidade La Salle em Canoas, RS, Brasil.

\section{Endereço para correspondência}

Laura D. Guerim

Universidade La Salle

Av. Victor Barreto, 2288, Prédio 8, Secretaria de Pesquisa e Pós-Graduação

Centro, 92010000

Canoas, RS, Brasil 